\title{
Influence of preliminary irradiation on radiation hardness of silicon and indium antimonide
}

\author{
P.G. Litovchenko ${ }^{1}$, W. Wahl ${ }^{2}$, A.A. Groza ${ }^{1}$, A.P. Dolgolenko ${ }^{1}$, A.Ya. Karpenko', \\ V.I. Khivrych ${ }^{1}$, O.P. Litovchenko' ${ }^{1}$, V.F. Lastovetsky ${ }^{1}$, V.I. Sugakov ${ }^{1}$ and V.K. Dubovy ${ }^{1}$ \\ ${ }^{1 . S C}$ Institute for Nuclear Research of NASU, Prospect Nauki 47, 03028, Kiev, Ukraine. \\ ${ }^{2}$ GSF, Institute of Radiation Protection, 85764 Neuherberg, Germany
}

\begin{abstract}
Radiation hardness of semiconductor detectors based on silicon is, first of all, determined by an introduction rate of point defects and aggregation of defect clusters. So introduction of electrically inactive impurity of oxygen promotes taking a vacancy stream aside from a doping impurity of phosphorus. Thus, in spite of the greater capture radius of vacancies by phosphorus atoms, high concentration of oxygen will suppress formation of Ecentres. Use of neutron transmutation doping method allows to receive silicon with enhanced radiation hardness. The preliminary irradiation with neutrons or charged particles with subsequent annealing also allows to increase radiation hardness of material. It is due to introduction into the volume of material some additional sinks for primary radiation defects that causes enhanced radiation hardness of such material.
\end{abstract}

Keywords: neutron, charged particles, silicon, indium antimonide, vacancy, interstitials, clusters of defects, radiation defects.

Paper received 26.04.01; revised manuscript received 14.05.01; accepted for publication 15.05.01.

\section{Introduction}

The problem of enhancing semiconductor radiation hardness is bound to the necessity of semiconductor devices long-term operation in nuclear radiation fields. For example, in a series of experiments at CERN with beams of charged particles, the detecting system consists of many different detectors. Their cost reaches many millions of US dollars. It is clear that the increase of their long-term stability will essentially lower the expenses for carrying out such expensive experiments. The situation with usage of magnetic sensors, based on semiconductor materials $A_{3} B_{5}$ at charged particle accelerators is similar. That's why radiation hardness of semiconductor materials and devices based on them was always in the focus of researchers attention.

\section{Methods of increasing semiconductor materi- als radiation hardness}

As it is known, during irradiation of semiconductors, wide spectra of defects are formed: point defects of vacancy and interstitial types, their complexes with impurities and clusters of defects. And while point defects are rather mobile under experimental conditions, clusters are immobile. There is a space charge around clusters, it may essentially effect the characteristics of materials and devices based on them.

The larger part of electrically active defects induced under irradiation are the impurity defect complexes. So, during $\gamma$-irradiation, silicon vacancies and interstitial atoms interacting with the impurity atoms and with each other, participate in formation of A-centers (V-O); E-centres $(\mathrm{P}-\mathrm{V})$; divacancies $(\mathrm{V}-\mathrm{V})$ and di-interstitials $(\mathrm{I}-\mathrm{I})$. These defects are efficient scattering and recombination centers for charge carriers in semiconductors.

In addition to point defects, high-energy nuclear particles and fast neutrons of a reactor create defect clusters surrounded with a space charge blocking a current of charge carriers. Radiation hardness of silicon semiconductor detectors is first of all determined by a rate of introduction of point defects and aggregation of defects into clusters.

In [1-3] the influence of various impurities, such as $\mathrm{B}, \mathrm{O}$, isovalent impurities $\mathrm{Sn}, \mathrm{C}$, Ge etc., on radiation hardness of silicon were studied.

Below we shall discuss some general methods of radiation increasing silicon hardness. Briefly, it is possible to extend the influence of various impurities on radiation 
hardness and reasons increasing it. So, the introduction of electrically inactive impurity of oxygen promotes the decrease of a vacancy stream from a doping phosphorus impurity. It is also known that the probability of phosphorus capture by vacancy is approximately two orders higher than for oxygen. Thus, in spite of the greater radius of capture of vacancies by phosphorus atoms, high concentration of oxygen will suppress the formation of E-centres. As detectors operate at room temperature, and hence, the Fermi level is close to the middle of a forbidden gap in high-resistivity $\mathrm{Si}$, though the level of A-centre $\left(E_{C}-0.164 \mathrm{eV}\right)$ will not influence on removal of carriers from a conduction band. Compared to phosphorous, boron has larger radius of vacancy capture, which rises radiation hardness of silicon highly doped with boron [1]. Interstitial atoms of silicon displace boron in an interstitial position, where boron is efficiently captured by divacancies or recombines with vacancies.

The temperature of the sample during irradiation also efficiently influences on formation of radiation defects. So the irradiation at temperature lower than $120 \mathrm{~K}$ reduces a removal rate of carriers, if measured at room temperature, by tens of times [4]. In [5] shown is that intensity of near - edge absorption and rate of postradiation annealing of disordered regions are much higher in the crystals of silicon irradiated by neutrons at $100 \mathrm{~K}$. It can be connected with high rate of introduction of nonoriented divacancies that are intensively annealed at temperatures above $140 \mathrm{~K}$. The low-temperature irradiation also suppresses participation of oxygen in formation of electrically active defects containing oxygen. That is one method more for increasing the semiconductor material radiation hardness.

In the course of silicon doping with isovalent impurities $\mathrm{Sn}$ and $\mathrm{Ge}$, the mechanical fields of strains caused by their large covalent radius are formed around the places of localization of these impurities. These fields of deformation strains may serve as sinks for vacancies and interstitial atoms, which causes suppression of vacancy complexes and divacancies formation. At high concentrations of these impurities in silicon $\left(>10^{18} \mathrm{~cm}^{-3}\right)$, a carrier removal rate measured at room temperature is suppressed. So, silicon doping with Ge suppresses the introduction rate of E- centres and divacancies by two orders, and Sn three times more effectively interacts with vacancies than oxygen. However, such large concentration of impurities (Sn, Ge) may result in material homogeneity deterioration and decreasing of charge carrier lifetime, which determines quality of Si detectors.

Dislocations, free from the impurity atmospheres, are also capable to interact efficiently with radiation defects $[6,7]$. At density $10^{6} \mathrm{~cm}^{-2}$ the dislocations in Si create deformation fields, under the influence of which vacancies and interstitial atoms generated by irradiation can migrate. Thus, radiation defects can lead to reorganization of a dislocation structure. But their small quantity and a high-temperature method of their generation interfere their use as efficient method for increasing detector material radiation hardness.
Growing microdefects (A, B, D) also efficiently interact with radiation defects. It is known, that when silicon single crystals are grown with small velocity $(\leq 3 \mathrm{~mm} / \mathrm{min})$, excess atoms of silicon in the range of crystallization are observed, so A-microdefects are formed. They are dislocation loops of interstitial type, deposited in a planes (110) and (111) and strongly directed along the direction $\{110\}$. They are easily determined by the selective etching and usually have the size (1-3) $10^{-4} \mathrm{~cm}$ at concentration $\left(10^{4}-10^{6}\right) \mathrm{cm}^{-3}$. The elastic deformation fields around A-microdefects may influence on radiation defects at distances no more than five diameters of a loop, as the electrostatic energy decreases as cube of distance.

At mean velocities of growing $(\sim 4 \mathrm{~mm} / \mathrm{min})$, Bmicrodefects of interstitial type are formed, representing flat aggregations in the shape of quadrates or rhombi deposited in planes (100) with sizes (200-500) A and concentrations $\left(10^{6}-10^{8}\right) \mathrm{cm}^{-3}$.

In the crystals grown up with high velocity $(>5 \mathrm{~mm} / \mathrm{min})$, when a deficit of silicon atoms is created in the crystallization range, D-microdefects of vacancy type, with sizes (40-60) A and concentrations of $10^{12} \mathrm{~cm}^{-3}$ are observed. D-defects are centres of capture for $\mathrm{Li}+$ atoms at their drift during the production of $\mathrm{Si}(\mathrm{Li})$ detectors. Routinely after annealing of neutron-doped silicon, used for creation of detectors, the formation of all types of microdefects sharply increases. Therefore, the method of gettering, offered in [8], and based on using a phosphoric glass $\left(\mathrm{POCl}_{3}\right)$, appears to be useful for NTD Si. Getter covering a surface of silicon generates interstitial atoms in the crystal bulk, and, as a consequence, D-microdefects are destroyed.

An increase of radiation hardness of the neutron-doped silicon (NTD Si) is also found in [9-11].

\section{Experiment and discussion of results}

Given below are experimental results concerning NTD $\mathrm{Si}$ and $\mathrm{a}$ method of a preliminary irradiation and annealing for increasing radiation hardness of silicon as well as indium antimonide.

\subsection{Preliminary neutron irradiation influence on radiation hardness of silicon in accordance with the results of optical investigations.}

Infrared absorption study of $\mathrm{Si}$ is an effective research technique for researching radiation effects due to increasing transmission of crystals in the range of wavelengths behind intrinsic band edge absorption. This fact is caused by decreasing majority carrier concentration in accordance with compensative activity of radiation defects. According to [12] near edge absorption after the irradiation with large doses of neutrons $\left(\sim 10^{19} \mathrm{n} / \mathrm{cm}^{2}\right)$ is caused by aggregations of vacancy and interstitial types. These are annealed in the interval of temperatures $400-500^{\circ} \mathrm{C}$. Decay of interstitial aggregations is accompanied by formation of complexes of interstitial atoms with oxygen. 


\section{P.G. Litovchenko et al.: Influence of preliminary irradiation on radiation hardness ...}

The occurrence of the whole spectrum of narrow bands of an oscillatory nature testifies it on a background of twophonon absorption. There is a full annealing of these defects at $700{ }^{\circ} \mathrm{C}$.

At repeated irradiation of «oxygen» samples the annealing gives much smaller formation of oxygen complexes. It is probably due to the fact that interstitial atoms instead of formation of complexes with oxygen prove to be more effective sinks than the dissolved atoms of oxygen. That's why, the silicon with the great content of oxygen at the repeated irradiation by neutrons and annealing behaves as low oxygen material [13].

Above-mentioned results show the influence of the repeated irradiation mainly on the processes of radiation defects annealing.

Certainly, we are more interested in the influence of preliminary irradiation on silicon radiation hardness.

We have carried out the test of the influence of various radiation treatments with subsequent annealing on FZ Si radiation hardness. The radiation treatments by fast neutrons $\left(\Phi=10^{17} \mathrm{n} / \mathrm{cm}^{2}\right)$, by thermal neutrons, in combination with fast and thermal neutrons, and also thermal neutrons with g quanta were used as the preliminary ones. After such treatments, all samples including the initial ones were annealed for two hours at $800^{\circ} \mathrm{C}$. Then samples were irradiated with fast neutrons by fluences $10^{16} \mathrm{n} / \mathrm{cm}^{2}$ at the research reactor WWR-M.

After irradiation the investigations were carried out by the method of infrared spectroscopy using spectrophotometers UR-20 and KSVU-23. The most sensitive to such irradiation is FZ $\mathrm{Si}$ in the spectral range near the edge of Si intrinsic absorption. The so-called near-edge absorption monotonously decreases with an increasing light wavelength. The selection absorption band with a maximum at $1.8 \mu \mathrm{m}$ that is related with divacancies is imposed on it. After irradiation with fluences of the order of $10^{16} \mathrm{n} / \mathrm{cm}^{2}$, the near-edge absorption in Si which is caused by disordered areas that are preferentially of vacancy type [12]. The investigations of absorption spectra in the spectral range $2-1.1 \mu \mathrm{m}$ on a large set of samples subjected to various combinations of preliminary radiation processings are carried out.

The results of these investigations are shown by the following two curves Fig. 1, where differential transmission spectra are given for clarity.

The curve $a$ in Fig. 1 represents the differential transmission spectrum of a reference Si sample not subjected to preliminary radiation treatment. It may be compared to a sample, preliminary irradiated with fast neutrons. Both samples were annealed and then were irradiated with neutron fluence $\Phi=10^{16} \mathrm{n} / \mathrm{cm}^{2}$.

The samples were cut off from one ingot. They had the identical thickness and the same surface treatment, and it is known, that preliminary $800^{\circ} \mathrm{C}$ annealing provides full annealing of the radiation defects fixed on infrared absorption, the apparent distinction in intensity of their transmission spectra should be attributed only due to preliminary radiation treatment by fast neutrons. As it follows from curve $a$, the intensity of absorption in

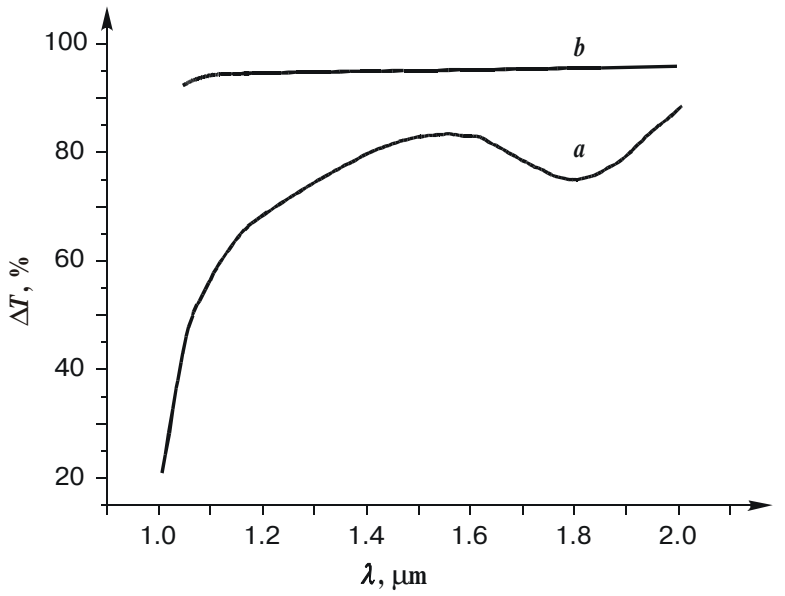

Fig. 1. Differential transmission spectrum dependence for Si samples after $800^{\circ} \mathrm{C}$ annealing and irradiation with neutron fluence $\Phi=10^{16} \mathrm{n} / \mathrm{cm}^{2}$ on wavelength $T=f(\lambda)$

$a$ - for the reference sample in comparison with a sample preliminarily irradiated by fast neutrons. Thickness of samples is $d=0.434 \mathrm{~cm}$.

$b$ - for a sample subjected to preliminary radiation treatment (by fast and thermal neutrons) in comparison with a sample preliminarily irradiated by gamma-quanta and thermal neutrons.

the reference sample is higher.

In Fig. 1, curve $b$ represents the differential transmission spectrum of two samples irradiated with fast neutrons $\Phi=10^{16} \mathrm{n} / \mathrm{cm}^{2}$ and preliminary subjected to various radiation treatments. As seen from this curve, the intensity of absorption in these samples does not depend on the method of preliminary radiation treatment used in the given experiment (an irradiation with fast neutrons, both thermal and fast neutrons, as well as gamma-quanta and thermal neutrons).

Thus, the given results evidently show that preliminary radiation treatment with fast or thermal neutrons yieldes in increasing radiation hardness due to formation of complex defects.

\subsection{The influence of preliminary irradiation with charged particles on radiation hardness of indium antimonide}

Let us consider the results of investigations concerning the efficiency of defect formation in preliminarily irradiated semiconductors using the example of indium antimonide irradiated by $47 \mathrm{MeV}$ protons.

As model, well studied and frequently used material we chose indium antimonide: sample 1 - not doped $n$-InSb with initial concentration of charge carriers $n_{80 \mathrm{~K}}=1.6 \cdot 10^{14} \mathrm{~cm}^{-3}$; sample $2-n$-InSb $<\mathrm{Sn}>$ with initial concentration of charge carriers $n_{80 \mathrm{~K}}=9 \cdot 10^{15} \mathrm{~cm}^{-3}$; sample $3-p$-InSb $<\mathrm{Cd}>$ with initial concentration of charge carriers $n_{80 \mathrm{~K}}=7 \cdot 10^{15} \mathrm{~cm}^{-3}$. It was decided to test a variant of introduction of sinks for radiation defects by 
preliminarily irradiation of a material with charged particles; i.e., a role of sinks, preliminarily put radiation defects should play. Samples were irradiated with $47 \mathrm{MeV}$ protons on isochronous cyclotron U-240 of KINR (Kyiv Institute for Nuclear Researches of the National Academy of Sciences of Ukraine) with beam intensity $(1-6) \times$ $\times 10^{12} \mathrm{~cm}^{-2} \mathrm{~s}^{-1}$ up to a certain fluence (preliminary irradiation), then irradiated samples were annealed at room temperature up to some intermediate state, and then the irradiation was recommenced (for sample 1 - at room temperature; for samples 2 and 3 - at $T=120 \mathrm{~K}$ ) with the purpose to compare efficiency of defect formation in samples at the intermediate state with defect formation in irradiated initial samples with the same characteristics, as they are at the samples in the intermediate state (preliminary irradiated and annealed). From Fig. 2 one can see that after recommencing irradiation the rate of radiation defects introduction into samples was sharply decreased. To our mind, it was possible due to the influence of sinks on newly induced radiation defects, as well as to radiation defects, induced by preliminary irradiation and annealing, which play the role of sinks. Analyzing the received result, it is possible to draw a conclusion that the combination of preliminary irradiation with annealing should establish the mode that may probably become a basis for radiation-thermal technology of increasing radiation hardness of semiconductor materials.

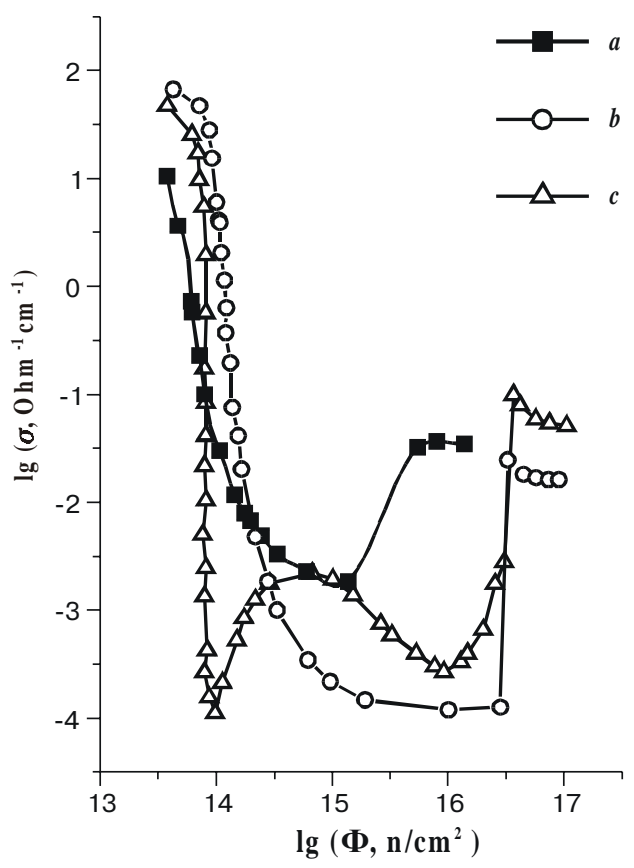

Fig. 2. Dose dependences of conductivity $\sigma_{80} \mathrm{~K}$ of indium antimonide irradiated by $47 \mathrm{MeV}$ protons:

$a-n$-InSb; irradiation at 100 and $300 \mathrm{~K}$;

$b-n-\mathrm{InSb}<\mathrm{Sn}>$; temperature of irradiation is $120 \mathrm{~K}$

$c-p$-InSb $<\mathrm{Cd}>$; temperature of irradiation is 120

\subsection{Neutron transmutation doping of silicon.}

As a matter of fact, the neutron transmutation doping of silicon, is one of variants of the raise of radiation hardness of silicon by the help of preliminary irradiation.

The neutron transmutation doping of silicon was carried out in a thermal column of a reactor WWR-M. Initial samples p-Si had a specific resistivity $\rho \sim 2-10 \mathrm{kOhm} \cdot \mathrm{cm}$. After irradiation by various doses of thermal neutrons and annealing at $850^{\circ} \mathrm{C}$ within 2 hours, samples of $\mathrm{p}-\mathrm{Si}$ with a specific resistivity $(12-40) \mathrm{kOhm} \cdot \mathrm{cm}$ and $\mathrm{n}-\mathrm{Si}$ with concentration of carriers $n=1.4 \cdot 10^{12} \mathrm{~cm}^{-3}$ were obtained.

Investigations of radiation hardness NTD silicon nand $p$-type are carried out at the irradiation by $\gamma$-quanta ${ }^{60} \mathrm{Co}$ and fast neutrons of the reactor with efficient energy $\left(E_{n} \sim 1 \mathrm{MeV}\right)$. In NTD silicon $n$-type conductivity carrier removal rate after neutron irradiation is much less than in control samples of silicon. In tables 1 and 2 carrier removal rate measured at room temperature for some radiation doses is shown. From tables 1 and 2, it is visible that NTD silicon $n$-type has higher radiation hardness to $\gamma$ radiation than to the neutron irradiation. So, at $\gamma$-an irradiation the carrier removal rate in NTD silicon more than one order less, than in a control specimen, and at a neutron irradiation removal rate is approximately two times less. In high-resistivity NTD $p-\mathrm{Si}(\rho \sim 10-40 \mathrm{kOhm} \cdot \mathrm{cm})$, it is not revealed apparent difference of radiation hardness compared to reference specimens of $p$-Si. Apparently, it is connected with a small radiation dose needed for obtaining high-resistivity $p$-Si and, as a consequence, by small concentration of sinks created by irradiation.

Fig. 3 shows the concentration of carriers in $n$-Si $\left(p_{\mathrm{o}}=1.76 \cdot 10^{12} \mathrm{n} \cdot \mathrm{cm}^{-3}\right)$, grown up by a $\mathrm{CZ}$ method as a function of $24 \mathrm{GeV}$ protons irradiation dose. The theoretical curves have been calculated using two following facts. $24 \mathrm{GeV}$ protons produce defects clusters with average radius $330 \mathrm{E}$ and rate of their introduction equal to $5 \cdot 10^{-3} \mathrm{~cm}^{-1}$. The position of the Fermi level concerning the conduction band on the average cluster is accepted as equal to $\left(E_{c}-0.5\right) \mathrm{eV}$. The curve $a$ was calculated in Gossic's model [14] without the account of introduction of point defects into conductive matrix $n$-Si. The curve $b$ describing the experimental value of carriers concentration takes into account the introduction of E-centres, divacancies and the defect levels $\left(E_{c}-0.49\right) \mathrm{eV}$ in view of their filling [15]. Fig. 3 shows that at least radiation hardness of $n$-Si can be improved twice using NTD Si, where less rate of introduction of point defects to the conductive matrix is significant in comparison to usual $\mathrm{Si}$.

The interstitial oxygen cannot only decrease the formation of E-centres. As is known, the oxygen in $\mathrm{Si}$ is effective recombination centre for primary radiation defects $(\mathrm{V}, \mathrm{I})$, that brings not only a space charge region of clusters, but also diminishes their average size in neutron irradiated n-Si. Fig. 4 shows the size distribution of cluster defects in $n$-Si created by fast neutrons of a reactor. The principles of calculation are explained in ref. [16]. Fig. 4 shows that oxygen suppresses formation of the largest defect clusters. 
P.G. Litovchenko et al.: Influence of preliminary irradiation on radiation hardness ...

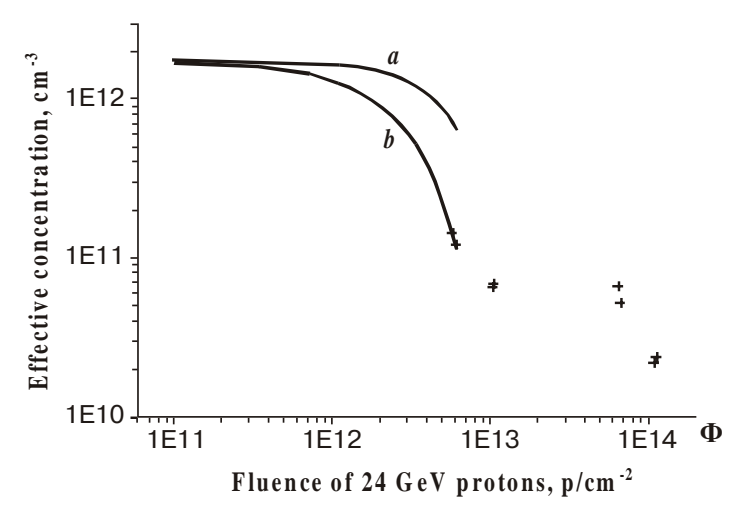

Fig. 3. Dose dependence of effective concentration of charge carriers in $n$-Si irradiated by $24 \mathrm{GeV}$ protons:

+ - experimental values;

$a$ - without any account of point defects;

$b$ - taking into account point defects.

When doping by neutrons, irradiation of crystals with thermal neutrons, fast neutrons and $\gamma$-quanta takes place, too. After neutron transmutation doping silicon and its subsequent annealing at $850^{\circ} \mathrm{C}$, defects of vacancy type combine and form electrically inactive vacancy complexes of silicon, and defects of an interstitial type under annealing are transmuted into complexes of an interstitial type (such microdefects as A, B and D).

Because of crystal lattice distortions around such aggregations, there are elastic stress fields of squeezing. Under action of these fields, vacancies generated by radiation and interstitial atoms migrate to aggregations, where they may annihilate or form complexes with each other (for example - divacancies). For this reason, concentration of initial vacancies, capable to participate in

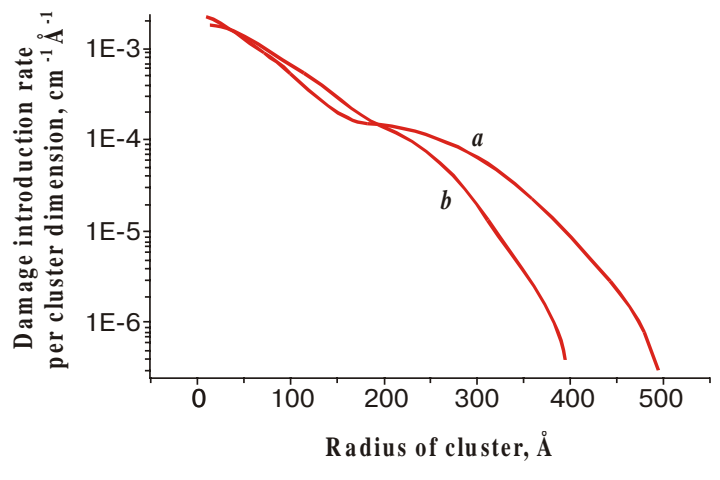

Fig. 4. Size distribution of defect clusters created by fast neutrons in $n$-Si grown by:

$a-F Z$ or $b-\mathrm{CZ}$ methods.

formation of complexes with the impurity atoms in the crystal volume, appears to be much less than in an FZ material. It yields in smaller efficiency of E-centre formation in NTD silicon.

Impurity-defect aggregations may also consist from electric active complexes $\mathrm{C}_{i}-\mathrm{C}_{s}$. Except for them aggregations is entered with own interstitials, interstitial carbon $\mathrm{C}_{2}$ or their associations, which in accord with experiment requirements are electrically neutral. By estimates, the mean radius of area is about $\sim 1.3 \cdot 10^{-4} \mathrm{~cm}$.

Naturally, the radiation hardness of NTD silicon will depend also both on concentration of sinks and that of phosphorus injected by transmutation. And for the best effect, it is necessary to have their optimum relation in each concrete case. Thus, received by us NTD n-type

Table 1. $\gamma$-irradiation

\begin{tabular}{|c|c|c|c|c|}
\hline \multirow{2}{*}{$\begin{array}{l}\text { Fluence } \\
{ }^{60} \mathrm{Co}, \mathrm{cm}^{-2}\end{array}$} & \multicolumn{2}{|c|}{ NTD } & \multicolumn{2}{|c|}{ Reference sample } \\
\hline & concentration $N, \mathrm{~cm}^{-3}$ & removal rate $\Delta N / \Phi, \mathrm{cm}^{-1}$ & concentration $N, \mathrm{~cm}^{-3}$ & removal rate $\Delta N / \Phi, \mathrm{cm}^{-1}$ \\
\hline 0 & $1.4 \cdot 10^{12}$ & - & $3.4 \cdot 10^{12}$ & - \\
\hline $1.6 \cdot 10^{14}$ & $1.4 \cdot 10^{12}$ & 0 & $2.67 \cdot 10^{12}$ & $4.5 \cdot 10^{-3}$ \\
\hline $4.8 \cdot 10^{14}$ & $1.33 \cdot 10^{12}$ & $1.5 \cdot 10^{-4}$ & $2.16 \cdot 10^{12}$ & $2.6 \cdot 10^{-3}$ \\
\hline $1.6 \cdot 10^{15}$ & $9.8 \cdot 10^{11}$ & $2.6 \cdot 10^{-4}$ & $1.7 \cdot 10^{12}$ & $1.1 \cdot 10^{-3}$ \\
\hline
\end{tabular}

Table 2. Irradiation by fast neutrons

\begin{tabular}{|c|c|c|c|c|}
\hline \multirow{2}{*}{$\begin{array}{l}\text { Fluence of } \\
\text { fast neutrons, } \\
n / \mathrm{cm}^{2}\end{array}$} & \multicolumn{2}{|c|}{ NTD } & \multicolumn{2}{|c|}{ Reference sample } \\
\hline & $\begin{array}{l}\text { concentration } \\
\qquad N, \mathrm{~cm}^{-3}\end{array}$ & $\begin{array}{r}\text { removal rate } \\
\Delta N / \Phi, \mathrm{cm}^{-1}\end{array}$ & $\begin{array}{c}\text { concentration } \\
\qquad N, \mathrm{~cm}^{-3}\end{array}$ & $\begin{array}{c}\text { removal rate } \\
\Delta n / \Phi, \mathrm{cm}^{-1} \mathrm{n} \cdot \mathrm{cm}^{-2}\end{array}$ \\
\hline 0 & $1.4 \cdot 10^{12}$ & - & $1.0 \cdot 10^{13}$ & - \\
\hline $4.6 \cdot 10^{11}$ & $1.1 \cdot 10^{12}$ & 0.66 & $9.2 \cdot 10^{12}$ & 1.74 \\
\hline $9.6 \cdot 10^{11}$ & $6.4 \cdot 10^{11}$ & 0.79 & $8.5 \cdot 10^{12}$ & 1.56 \\
\hline
\end{tabular}




\section{P.G. Litovchenko et al.: Influence of preliminary irradiation on radiation hardness ...}

silicon for manufacturing detectors of nuclear radiations is more stable to $\gamma$-radiation of $\mathrm{Co}^{60}$ and fast neutrons. It is caused by activity of high concentration of sinks having different nature and generated during a nuclear doping as well as post-treatments and annealing.

\section{Conclusion}

The analysis of the given data concerning the method of a preliminary irradiation enables to draw a conclusion about the possibility to create using this method new radiation - thermal technology to increase semiconductor materials radiation hardness.

Therefore, the most promising is the method of preliminary irradiation offered above and annealing including neutron doping, due to an increased concentration of sinks for defects.

This method is applicable for increasing radiation hardness of many semiconductors, but is the most perspective for silicon that has been used in developing precise detectors and other semiconductor sensors working in fields of nuclear radiations.

\section{References}

1. J. Matheson, M.S. Robbins and S.J. Watts, The effect of radiation induced defects on the performance of high resistivity silicon diodes // RD 20 Technical Report CERN RD20/ TN 95-36 (1995).

2. B. Dezillie, S. Betes, M. Glaser, F. Lemeilleur, C. Leroy, Nucl. Instr. And Meth. In Phys. Research A 388, pp. 314-317 (1997), International Conf. On Radiation Effects on Semiconductor Materials, Detectors and Devices, Florence, Italy, 6-8 March 1996.

3. A.G. Italiantsev, A.I. Kurbanov, V.N. Mordkovich, E.E. Rubinova, E.M. Temper, V.A. Trunov, Influence of neutron irradiation and annealing on $\mathrm{Si}$, doped with $\mathrm{Ge}$, properties / / Fiz. Tekhn. Polupr., 22 (5) p. 834-838 (1988).

4. K.L. Starostin, Temperature dependence of concentration decreasing rate in $n$-Ge and $n$-Si under fast neutron irradiation // Fiz. Tekhn. Polupr., 4(9) p. 1823-1824 (1970).
5. G.S. Karumidze, Temperature irradiation influence with neutrons on defects of structure formation in CZ Si // Fiz. Tekhn. Polupr., 24(11) p. 1973-1977 (1990).

6. L.A. Kazakevich, V.I. Kuznetsov, P.F. Lugakov, Regions of radiation defects aggregation formation in dislocation $\mathrm{Si} / /$ Fiz. Tekhn. Polupr., 22(3) p. 499-502 (1988).

7. L.A. Kazakevich, P.F. Lugakov, I.M. Filipov, Charge carriers recombination at dislocation and radiation defects in p-Si // Fiz. Tekhn. Polupr., 23(4) p. $722-725$ (1989).

8. Knowlton, J.T. Walton, J.S. Lee, Y.K. Wong, E.E. Haller, W. Von. Ammon, W. Zulehner, Lithium-Ion Drifting: Application to the study of Point Defects in Floating-Zone Silicon // Proceedings of the 18th International Conf. On Defects in Semiconductors, M. Suezawa and H. Katayama-Yoshida, Editors, p. 1776. Material Science Forum vol. 196-201, Trans. Tech. Publications, Switzerland, 1995.

9. P.F. Lugakov, V.V. Lukjanitsa, The defects' nature and peculiar properties of its creation during neutron transmutation doped silicon irradiation // Fiz. Tekhn. Polupr., 17(9) p. 1601 (1983).

10. A.P. Didkovsky, A.K. Saakova, V.I. Khivrich, Some electrical properties of high resistivity radiation-doped silicon // Fiz. Tekhn. Polupr. 10(3), p. 543 (1976).

11. I.I. Kolkovsky, L.F. Lugakov, V.V. Shusha, Recombination properties of radiation defects in NTD Si // Fiz. Tekhn. Polupr., 20(5) 964-967 (1986).

12. A.A. Groza and V.I. Hivrich, Near edge adsorption in the silicon irradiated by neutrons and by $1.5 \mathrm{MeV}$ electrons // Phys. Tech. Poluprovodnikov 13(5), pp. 870-874 (1979).

13. A. A. Groza, V. I. Kutz, P. G. Litovchenko and V. I. Khivrich, The factors influencing formation oxygen - defect complexes in silicon single crystals // Elektronnaya Technika v.6, 5(178), pp. 60-61 (1983).

14. B. R. Gossick, Disordered Regions in Semiconductors // J. Appl. Phys. 30, pp. 1214-1218 (1959).

15. P.G. Litovchenko, F. Lemeilleur, A.P. Dolgolenko, L.I. Barabash, N.N. Kolychev, A.P. Litovchenko, V.F. Lastovezky, T.I. Kibkalo, L.A. Polivtsev, Dose dependence of the concentration of carriers in high resistivity Si irradiated by $24 \mathrm{GeV}$ protons and properties of the detectors on its base, // DESYProceedings-1998-02 February 1998. 3rd. ROSE Workshop on Radiation Hardening of Silicon Detectors DESY Hamburg, 12-14 February 1998.

16. A.P. Dolgolenko and I.I. Fishchuk, Defect Clusters and Simple Defect Build-Up Kinetics in Fast-Neutron Irradiated $n$ Si // Phys. Stat. Sol. A 50, p.751 (1978). 